\title{
An investigation of the appearance of a long range nuclear potential in
} ultra low energy nuclear synthesis

\author{
S. Oryu ${ }^{1 \star}$, T. Watanabe ${ }^{1}$, Y. Hiratsuka ${ }^{2}$ and M. Takeda ${ }^{3}$ \\ 1 Department of Physics, Faculty of Science and Technology, \\ Tokyo University of Science, Noda, Chiba 278-8510, Japan \\ 2 Preparation School of HLF Ltd, Kiryu, Gunma 376-0021, Japan \\ 3 Department of Information Science, Faculty of Science and Technology, \\ Tokyo University of Science, Noda, Chiba 278-8510, Japan \\ * oryu@rs.noda.tus.ac.jp
}

October 30,2019 Surrey, UK, 2-6 September 2019

\begin{abstract}
A new potential, the so called "GPT", represents a Yukawa-type potential for shorter range, but a $1 / r^{n}$-type potential for longer range where $n=2$ includes the Efimov-like potential. In order to confirm the existence of a GPT potential, we investigate the possibility of creating the lanthanum (La)-nucleus via the ultra low energy reaction: $\operatorname{Cs}(2 \mathrm{~d}, \gamma) \mathrm{La}$ on the three-ion quasi-molecule $\mathrm{CsD}_{2}$ in a $\mathrm{CsD}_{2} \mathrm{Pd}_{12}$-cluster. Here, the $\mathrm{D}-\mathrm{Cs}-\mathrm{D}$ (or d-Cs-d) three-body bound state and wave function are used to calculate the electro-magnetic (EM) transition from the molecular states to the nuclear states in the $\mathrm{Cs}(2 \mathrm{~d}, \gamma)$ La reaction. We obtain an approximate E2-transition ratio between the $1 / r^{2}$-type long range potential in combination with the short range nuclear potential and the short range nuclear potentials of $W_{i \rightarrow f}^{E 2^{\prime} ; L} / W_{i \rightarrow f}^{E 2^{\prime} ; S} \approx 10^{8}$ which implies that the quasi molecular state is stable for the usual transition $W_{i \rightarrow f}^{E 2^{\prime} ; S}$, but nuclear synthesis occurs for $W_{i \rightarrow f}^{E 2^{\prime} ; L}$ with long range. We conclude that if the reaction $\operatorname{Cs}(2 \mathrm{~d}, \gamma) \mathrm{La}$ occurs, then the existence of the GPT potential is confirmed.
\end{abstract}

\section{Introduction}

Based on three-body kinematics, we proposed a general particle transfer (GPT) potential which appears, not only at the three-body break-up threshold (3BT) but also at the quasi two-body threshold (Q2T) [1] [2]. The "GPT-potential" is a Yukawa-type potential for shorter range, but a $1 / r^{n}$-type potential for longer range, where $n=2$ includes the Efimovlike potential [3] [4] [5]. We briefly review the GPT potential in section 2. The GPT potential could generally exist in many systems, from atomic-molecular physics to hadron physics, especially in the threshold behaviors where the two-body interaction becomes very small.

In this paper, we explore a subject named the "ultra low energy nuclear synthesis" in section 3. In the traditional nuclear fusion approach, the most serious problem is whether the incident energy is sufficient to penetrate the Coulomb barrier, because the reaction is usually started in the free field. We point out that the Cs-d penetration in the quasimolecule, which is developed in the Pd-crystal, could easily occur [6], since the energy 
levels of such a quasi-molecule could exist close to the top of the Cs-d Coulomb barrier or over the barrier in the "Pd-cage", which is a repulsive Coulomb barrier between the ions (Cs/D) and outer Pd-cage. Experimental results were published in 2002 by Iwamura et al. [7], and Hioki et al. in 2013 [8]. The most critical point, whether the nuclear synthesis in their experiments did occur, will be discussed in our theoretical view in section 4 .

\section{The general particle transfer potential}

In the three-body AGS equations, the coincidence of singularities in the kernel arises between the particle transfer term and the propagator of the two-body sub-system [9] which brings about a serious problem at the 3BT. On the other hand, in the quasi two-body scattering problem, where the two-body subsystem has a bound state in the three-body problem, the kernel also diverges in the particle transfer diagram and the two-body Green's function at the Q2T. It should be noted that the 3BT corresponds to the case of two-body zero binding energy in the Q2T. Therefore, the Q2T is more general than the 3BT. Such a coincidence singularity is also found in the two-body Lippmann-Schwinger equation with the Coulomb potential which gives rise to a serious problem with the Green's function at the on-shell threshold [10]- [12]. In the Coulomb problem, such as the electron-proton system, the potential is given by

$$
V(r)=-e^{2} / r
$$

Efimov pointed out that the three-body bound states with the two-body scattering length of $a \rightarrow \infty$ accumulate on the 3BT [4] [5]. Historically, Nicholson pointed out in 1962 that such a scattering length is exactly given by another type of attractive long range potential in the two-body Lippmann-Schwinger equation [3],

$$
V(r)=-\alpha / r^{2},
$$

with a proper constant $\alpha>0$. Therefore, one should keep in mind that such long range potentials are closely related with the coincidence of singularities in the kernel of the twoand three-body scattering equations.

In 2012, we proposed the GPT potential, which is introduced at the Q2T in the threebody problem [1]. As mentioned above, since the Q2T singularity is more practical than the 3BT case, we do not mind the "infinite value of the scattering length" for the two-body potential which was discussed by Efimov [4], although the Q2T persists even in the case of the two-body "finite value of the scattering length" for the binding energy $\epsilon_{B} \neq 0$.

Let us recall our theory briefly at the Q2T, the Born term of the AGS equation is given in terms of two-body form factors with different channels $g_{\alpha}(p), g_{\beta}\left(p^{\prime}\right)$ and two-body momenta $p, p^{\prime}$, and $\nu, \mu$ for the reduced masses between two particles, and between a spectator and the two-body mass, and also with the three-body free energy $E$,

$$
Z_{\alpha \beta}\left(q, q^{\prime} ; E\right)=\frac{g_{\alpha}(p) g_{\beta}\left(p^{\prime}\right)\left(1-\delta_{\alpha \beta}\right)}{E-q^{2} / 2 \mu-p^{2} / 2 \nu} \rightarrow \frac{g_{\alpha}(p) g_{\beta}\left(p^{\prime}\right)\left(1-\delta_{\alpha \beta}\right)}{E-q^{2} / 2 \mu+\epsilon_{B}},
$$

For the Q2T: $E_{c m} \equiv E+\epsilon_{B}=0, q=0$, and Eq.(3) becomes

$$
Z_{\alpha \beta}\left(q, q^{\prime} ; E\right) \rightarrow \infty .
$$

On the other hand, at the Q2T, the two-body bound state becomes $\epsilon_{B} \neq 0$, and therefore, the Efimov criterion : $a \rightarrow \infty$ is not satisfied anymore. The propagator at the 
Q2T becomes with the on-shell relation $z=E-q^{2} / 2 \mu(\leq 0)$,

$$
\tau_{B}(z)=\frac{f(z)}{\epsilon_{B}+z}=\frac{f(z)}{\left(\epsilon_{B}+E\right)-q^{2} / 2 \mu} \rightarrow \infty .
$$

Therefore, Eq.(4) and Eq.(5) indicate the "singularity coincidence" at the Q2T, where one could imagine "an existence of long range potential".

Just below the Q2T $\left(E_{c m} \leq 0\right)$, the AGS-Born term [9] becomes with $p=p^{\prime}=0$,

$$
Z_{\alpha \beta}\left(q, q^{\prime} ; E\right)=\frac{g_{\alpha}(0) g_{\beta}(0)\left(1-\delta_{\alpha \beta}\right)}{-\left|E_{c m}\right|-q^{2} / 2 \mu}=-\frac{C_{\alpha \beta}}{q^{2}+\sigma^{2}},
$$

where $C_{\alpha \beta}=2 \mu g_{\alpha}(0) g_{\beta}(0)\left(1-\delta_{\alpha \beta}\right)$, and $\sigma=\sqrt{2 \mu\left|E_{c m}\right|}$. Therefore, the Fourier transformation of this energy dependent potential becomes,

$$
\mathcal{F}\left[Z_{\alpha \beta}\left(q, q^{\prime} ; E\right)\right]=-\mathcal{F}\left[\frac{C_{\alpha \beta}}{q^{2}+\sigma^{2}}\right]=V_{0} \frac{e^{-\sigma r}}{r},
$$

with $V_{0}<0$. The $r$-space potential is a kind of Yukawa potential, but energy dependent. For $\sigma=0$ or $E_{c m}=0$, it becomes the Coulomb potential (or the gravitational potential); therefore, our AGS equation is essentially the same equation as the Coulomb LippmannSchwinger equation such as in electron-proton scattering except for the coupling constant [9]. In order to solve the eigenvalue equation with the energy dependent potential of Eq.(7), we have to solve it consistently with the two energies which are seen in the potential and in the eigenvalue. However, the method is very complicated and hard to obtain with good accuracy. Therefore, we introduced in ref. [1], an energy average by using a probability density function with respect to the possible energy range, which also represents effects of the structure or the form factors of the composite particles,

$$
P_{\sigma}=\frac{\sigma^{2 \gamma+1} e^{-a \sigma}}{\rho}
$$

with

$$
\rho=\int_{0}^{\infty} \sigma^{2 \gamma+1} e^{-a \sigma} d \sigma=\frac{\Gamma(2 \gamma+2)}{a^{2 \gamma+2}},
$$

where $e^{-a \sigma}$ is a damping factor with a range parameter $a$. By using the probability density function, the expectation value of the energy-dependent potential becomes energy independent. This transformation is called the "Euler integral of the second kind" with respect to Eq.(7), or a Laplace transformation with a weight function. Therefore, by using Eq.(8) and Eq.(9), Eq.(7) becomes,

$$
\begin{aligned}
\mathcal{L}\left\{\mathcal{F}\left[Z_{\alpha, \beta}\left(q, q^{\prime} ; E\right)\right]\right\} & =\mathcal{L}\left\{\frac{V_{0} e^{-\sigma r}}{r}\right\}=\frac{V_{0}}{\rho} \int_{0}^{\infty} \sigma^{2 \gamma+1} e^{-a \sigma} \frac{e^{-\sigma r}}{r} d \sigma \\
& =V_{0} \frac{a^{2 \gamma+2}}{r(r / 2+a)^{2 \gamma+2}} .
\end{aligned}
$$

Thus, the predicted GPT potential is obtained.

Therefore, it is seen that the GPT potential is a combination of a Yukawa-type potential at shorter range but $1 / r^{n}$-type potential for longer range. $\gamma=-1$ gives an attractive Coulomb or the gravitational-type potential for any region which is given by zero mass 
Table 1: The GPT potential $V_{0} a^{2 \gamma+2} /\left[r(r / 2+a)^{2 \gamma+2}\right]$ is illustrated, which is given by an energy average below the 3BT $\left(E=0, \epsilon_{B}=0\right)$ and below the Q2T $\left(E=-\epsilon_{B}, \epsilon_{B} \neq 0\right)$ with two-parameters $a$ and $\gamma$. The potential properties for the longer and shorter ranges are shown with respect to the parameter $\gamma . V_{0}(<0)$, a potential depth which is analytically given by Eq.(7).

\begin{tabular}{cccc}
\hline \hline$\gamma$ & short range potential $r \ll a$ & potential & long range potential $a \ll r$ \\
\hline-1 & $V_{0} / r$ & $V_{0} / r$ & $V_{0} / r$ \\
$-1 / 2$ & $V_{0} e^{-r / 2 a} / r$ & $V_{0}(2 a) /[r(r+2 a)]$ & $V_{0}(2 a) / r^{2}$ \\
0 & $V_{0} e^{-2 r / 2 a / r}$ & $V_{0}(2 a)^{2} /\left[r(r+2 a)^{2}\right]$ & $V_{0}(2 a)^{2} / r^{3}$ \\
$1 / 2$ & $V_{0} e^{-3 r / 2 a / r}$ & $V_{0}(2 a)^{3} /\left[r(r+2 a)^{3}\right]$ & $V_{0}(2 a)^{3} / r^{4}$ \\
1 & $V_{0} e^{-4 r / 2 a / r}$ & $V_{0}(2 a)^{4} /\left[r(r+2 a)^{4}\right]$ & $V_{0}(2 a)^{4} / r^{5}$ \\
$3 / 2$ & $V_{0} e^{-5 r / 2 a / r}$ & $V_{0}(2 a)^{5} /\left[r(r+2 a)^{5}\right]$ & $V_{0}(2 a)^{5} / r^{6}$ \\
2 & $V_{0} e^{-6 r / 2 a / r}$ & $V_{0}(2 a)^{6} /\left[r(r+2 a)^{6}\right]$ & $V_{0}(2 a)^{6} / r^{7}$ \\
$\cdot$ & $\ldots$ & $\cdots$ & $\cdots$ \\
. & $\ldots$ & $\cdots$ \\
\hline \hline
\end{tabular}

particle transfer. On the other hand, $\gamma=-1 / 2$ means the Efimov-type potential for the longer range. One could imagine that the mass of the transfered particle could be very small compared to the total mass of the parent particles. Therefore, an interaction between Cs-atom and D-atom by electron transfer could be given by the $1 / r^{2}$-type potential. While a one pion transfer potential between the Cs and D nuclei could be the $1 / r^{2}$-type or $1 / r^{3}$ type which should be added to the nuclear Cs-D Wood Saxon potential.

\section{An Investigation of the Long Range Force in the $\mathrm{Cs}(2 \mathrm{~d}, \gamma)$ La Reaction}

It is expected that many three-body systems such as unstable nuclei, nuclear halo systems and hypernuclei as well as nuclear forces could be affected by the GPT potential. Especially, the long range effect due to the GPT potential could appear in a kinematic region where the two-body potential becomes weak.

Recently, it was claimed that several incomprehensible phenomena were found in the condensed matter nuclear science field. In 2002, Iwamura et al. found that, when Cs is added on the surface of a $\mathrm{Pd}$ complex, $\mathrm{D}_{2}$ gas permeation at $343 \mathrm{~K}$ changes Cs to $\mathrm{Pr}$ which means that a reaction: $\mathrm{Cs}+2 \mathrm{D}_{2} \rightarrow \operatorname{Pr}$ occurs [7]. They also found that a reaction: $\mathrm{Sr}+2 \mathrm{D}_{2} \rightarrow$ Mo also occurs. Furthermore, Hioki et al. confirmed the previous reactions in 2013 [8].

Usually, in the free field, it is known that the molecular state is stable, and never changes to the nuclear state, because the molecular state is energetically too far from the nuclear state and the wave functions could not overlap one another, and also the Coulomb barrier can not be penetrated at room temperature.

However, in condensed matter, if and only if, some supplemental states generated by the long range nuclear potential could mediate a transition from the molecular state to nuclear states, could the nuclear synthesis occur as an electro-magnetic (EM) transition between both states. Finally, the molecular state: $\mathrm{CsD}_{4}$ could change to a Pr-nucleus which could be accomplished by the direct five-body transition $\mathrm{CsD}_{4} \rightarrow \mathrm{Pr}$, or by the sequential reaction $\mathrm{CsD}_{2} \rightarrow \mathrm{La}$ and $\mathrm{LaD}_{2} \rightarrow \operatorname{Pr}$.

In this paper, we first investigate the three-body reaction problem: $\mathrm{CsD}_{2} \rightarrow \mathrm{La}$ in the Pd complex instead of the five-body problem: $\mathrm{CsD}_{4} \rightarrow \operatorname{Pr}$, and compare two cases with our 
long range potential plus nuclear potential and without the long range potential. Therefore, this reaction could be represented by $\operatorname{Cs}\left(\mathrm{d}_{2}, \gamma\right) \mathrm{La}$ in nuclear reaction terminology in the $\mathrm{CsD}_{2} \mathrm{Pd}_{12}$ system with the shape of cub-octahedron [14]. Although, the Coulomb barrier still exists in this problem, we should say again that the barrier is not in the free field but in the Pd cluster (or Pd cage). For this reason, the energy levels can be calculated from the bottom of the ion-ion potential to the top of the Coulomb barrier. Therefore, the penetration problem is resolved in the quasi-molecule: $\mathrm{CsD}_{2}$ in the $\mathrm{CsD}_{2} \mathrm{Pd}_{12}$ system, and $\mathrm{CsD}_{4}$ in $\mathrm{CsD}_{4} \mathrm{Pd}_{12}$, and also $\mathrm{CsD}_{6}$ in the system $\mathrm{CsD}_{6} \mathrm{Pd}_{12}$ [13], [14].

In order to investigate a very shallow nuclear state, we would like to study the D-Cs-D three-ion problem by a high precision three-body variational (HPV) method with 80 to 100 digits of accuracy based on a usual variational approach. For the nuclear potentials Cs-D, D-D, the WS potential is adopted. The repulsive Coulomb potentials for Cs-D, D$\mathrm{D}$, and $(\mathrm{Cs} / \mathrm{D})-\mathrm{Pd}$ cage are taken into account, where the repulsive Coulomb (Cs/D)-Pd potentials are given by a one-body $V_{c}^{P d}$ potential. We also adopted a three-cluster force to fit the ground state of La: $V_{t}$. The two- or three-body long range hadron interactions are introduced by the GPT potential which are given by a kind of "three-body Efimov potential" [4]. Some parameters of the potential are chosen to cancel the three-body force effects at the tail of the WS potential [14], where the so-called "three-body Efimov potential" should not be confused with the two-body potential which produces the infinite value of the two-body scattering length.

The WS potential is given for the $i^{t h}-\mathrm{N}$ and $j^{t h}-\mathrm{N}$ nuclear potential: $V_{W}^{N_{i} N_{j}}\left(\mathrm{r}_{i j}\right)$,

$$
V_{W}^{N_{i} N_{j}}\left(r_{i j}\right)=V_{W 0}^{N_{i} N_{j}}\left[1+\exp \left(\frac{r_{i j}-R_{W}^{N_{i} N_{j}}}{a_{W}^{N_{i} N_{j}}}\right)\right]^{-1}
$$

with parameters, $V_{W 0}^{C s d}=-79.30 \mathrm{MeV}, \quad V_{W 0}^{d d}=-27.57 \mathrm{MeV}, \quad R_{W}^{C s d}=10.21 \mathrm{fm}, \quad R_{W}^{d d}=$ $1.49 \mathrm{fm}, \quad a_{W}^{C s d}=0.4 \mathrm{fm}, \quad a_{W}^{d d}=0.3 \mathrm{fm}$, respectively. Here, we used only the central part of the potential, and the non-central part of the WS potential has been omitted for the first time, because the highest nuclear energy level is not very sensitive to the non-central part. Furthermore, the EM transition from the molecular state to the highest nuclear energy level is very important for the nuclear synthesis. Therefore, we calculate only the S-state wave function in the beginning.

The ion-ion Coulomb potential is

$$
V_{c}^{N_{i} N_{j}}\left(r_{i j}\right)=\left\{\begin{array}{ccc}
\frac{Z_{i} Z_{j} e^{2}}{8 \pi R_{c}^{N_{i} N_{j}}}\left[3-\left(\frac{r_{i j}}{R_{c}^{N_{i} N_{j}}}\right)^{2}\right] & \text { for } \quad r_{i j} \leq R_{c}^{N_{i} N_{j}} \\
\frac{Z_{i} Z_{j} e^{2}}{4 \pi r_{i j}} & \text { for } \quad R_{c}^{N_{i} N_{j}}<r_{i j}
\end{array}\right.
$$

with $R_{c}^{C s d}=10.21 \mathrm{fm}$, and $R_{c}^{d d}=1.49 \mathrm{fm}$.

The $\mathrm{Pd}-\mathrm{N}_{i}$ Coulomb barrier is represented by a one-body potential,

$$
V_{c}^{P d N_{i}}\left(r_{i}\right)=V_{c 0}^{P d}\left(\frac{r_{i}}{a_{c}^{P d}}\right)^{10} \exp \left\{-\left(\frac{r_{i}-a_{c}^{P d}}{b_{c}^{P d}}\right)^{2}\right\}
$$

with $V_{c 0}^{P d}=1.0 \times 10^{-4} \mathrm{MeV}, \quad a_{c}^{P d}=5.0 \times 10^{5} \mathrm{fm}$, and $b_{c}^{P d}=3.1623 \times 10^{5} \mathrm{fm}$, respectively. These parameters for the $\mathrm{Pd}$-cage indicate that the location of $\mathrm{Pd}$ is $1.57 \times 10^{6} \mathrm{fm}$, and $2.73 \mathrm{MeV}$ height, however, these values may vary.

In this paper, we adopt a three-cluster (or body) potential as,

$$
V_{t}\left(r_{1}, r_{2}, r_{3}\right)=V_{t 0} \exp \left[-\left(\frac{r_{1}-r_{2}}{a_{t}}\right)^{2}-\left(\frac{r_{2}-r_{3}}{a_{t}}\right)^{2}-\left(\frac{r_{3}-r_{1}}{a_{t}}\right)^{2}\right],
$$


where $V_{t 0}=1800 \mathrm{MeV}$ and $a_{t}=3.0 \mathrm{fm}$ are used to fit the ground state of La by adding the Coulomb force.

We obtain a very good fit to the experimental ground state energy $\mathcal{E}_{0}=-32.3 \mathrm{MeV}$, the root mean square (rms) radius $R_{0}=6.25 \mathrm{fm}$, the highest excited energy $\mathcal{E}_{\max }=-3.5134$ $\times 10^{2} \mathrm{keV}$, for the usual nuclear potential by the HPV calculation. Therefore, the highest energy level is far from that of the usual molecular state.

On the other hand, in order to obtain shallow energy levels that could be close to the molecular states we adopt a kind of three-body long range potential with a $1 / r_{i j}^{n}$ tail which was proposed as the GPT potential [1], [2] with a Lorentz form or given by the modified three-body Efimov potential [4]:

$$
V_{e}\left(r_{1}, r_{2}, r_{3}\right)=V_{e 0} a_{e}^{n}\left[\left(r_{1}-r_{2}\right)^{n}+\left(r_{2}-r_{3}\right)^{n}+\left(r_{3}-r_{1}\right)^{n}+a_{e}^{n}\right]^{-1},
$$

where we choose $n=2$ in this paper. Parameters $V_{e 0}=-80000 \mathrm{MeV}$, and $a_{e}=5000 \mathrm{fm}$ are taken, and also a supplemental $a_{e}^{n}$ is used to avoid divergence. It could be noticed that these parameters seem to be too large to compare to the parameters in the WS potential, however Eq.(15) does not change the usual nuclear potential in the shorter range together with the three-body potential.

On the $k$-particle transfer diagram in the AGS Born term [9], there are two types in Eq.(15) for $n=2$. One of them is the "two-body long range type" which appears in the two-body form factor, and another one is the "three-body long range type" which appears between parent particles.

A) The three-body long range potentials between parent particles are given by

$$
\begin{array}{ll}
V_{e}=V_{e 0} a_{e}^{2} /\left[r_{i j}^{2}+a_{e}^{2}\right] & \left(\text { for } r_{k i}=0 \quad \text { and } r_{j k}=0\right), \\
V_{e}=V_{e 0} a_{e}^{2} /\left[2 r_{i j}^{2}+a_{e}^{2}\right] & \left(\text { for } r_{k i}=0 \quad \text { or } r_{j k}=0\right) .
\end{array}
$$

B) The two-body long range potentials are represented by

$$
V_{e}=V_{e 0} a_{e}^{2} /\left[2 r_{k j}^{2}+a_{e}^{2}\right]=V_{e 0} a_{e}^{2} /\left[2 r_{k i}^{2}+a_{e}^{2}\right] \quad\left(\text { for } \quad r_{i j}=0 \quad\right) .
$$

C) Putting $\mathrm{r}_{k}=\left(\mathrm{r}_{i}+\mathrm{r}_{j}\right) / 2$ for the fixed $k$-particle, Eq.(15) becomes

$$
V_{e}=V_{e 0} a_{e}^{2} /\left[3 r_{i j}^{2} / 2+a_{e}^{2}\right]
$$

Eq.(19) represents another type of three-body long range potential of (A). The three-body force long range potential of Eq.(15) indicates the non-linearity of the GPT potential which gives not only two-body long range but also three-body long range.

The highest three-cluster (or -ion) nuclear level can be well represented by the S-wave or even central potential. While, the La ground state was adjusted by using the central two-cluster Cs-d and d-d potentials to obtain $-32.3 \mathrm{MeV}$ with the three-cluster force in Eq.(14). We confirmed that the phenomenological method for the La ground state is not very sensitive to the shallow bound states.

The nuclear interaction usually appears in the region of $0 \leq r<10^{3} \mathrm{fm}$, however, the molecular levels have been calculated in the region of $10^{4} \mathrm{fm} \leq r<10^{6} \mathrm{fm}$. Therefore, it was historically thought that the nuclear state wave function and the molecular state wave function do not overlap to give rise to the EM transition. This means that the molecular state cannot transfer to the nuclear state. However, the binding energy and wave function of $\mathrm{CsD}_{2}$ in the $\mathrm{Pd}_{12}$-cage are completely different from the free system. We solved the three-ion system in the $\operatorname{Pd}_{12}$-cage for the full region: $0 \leq r<\infty$ by the HPV method. In other words, for the nuclear and the molecular systems we can solve the eigen-equation on 


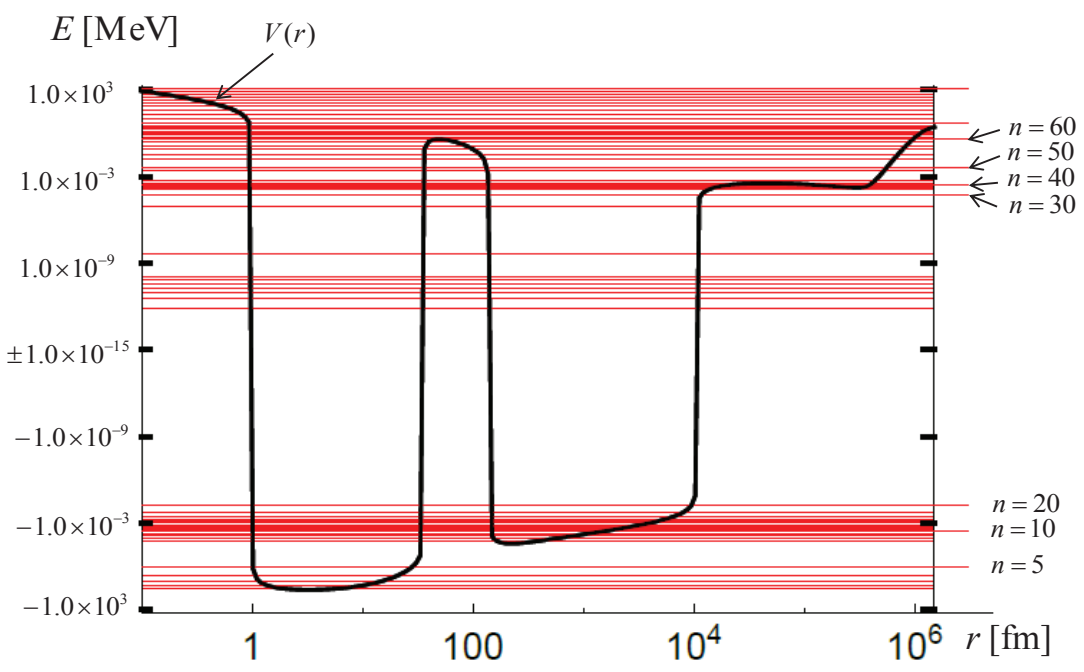

Figure 1: Three-body energy levels and potential "with the long range potential". The attractive potential part of the shorter range (fm region) indicates mainly the nuclear WS potential, and the next attractive part of $10^{3} \mathrm{fm}$ region comes from mainly the GPT potential, and the final hollow in the $10^{5} \mathrm{fm}$ region is the Coulomb attractive potential by the $\mathrm{Cs}$ ion and the $\mathrm{Pd}$ cage. $n$ is the principal quantum number. $1 \leq n \leq 5$ shows the La nuclear levels, $6 \leq n \leq 20$ are special levels by the GPT potential which belong to the nuclear excited states, and $21 \leq n \leq 60$ states represent the molecular levels for $\mathrm{CsD}_{2}$, respectively. 


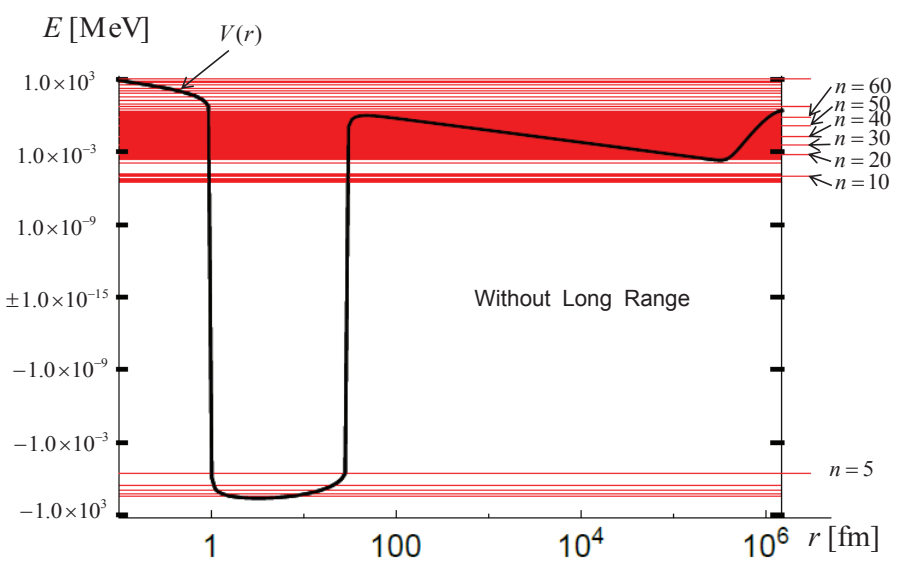

Figure 2: Energy levels and potential "without long range potential". The attractive potential part of the shorter range (fm region) indicates mainly the nuclear WS potential, and the next attractive part in the $10^{5} \mathrm{fm}$ region is the Coulomb attractive potential between the Cs ion and the Pd cage. $n$ is the principal quantum number. $1 \leq n \leq 5$ shows the La nuclear levels, and $6 \leq n \leq 60$ states represent the molecular levels for $\mathrm{CsD}_{2}$, respectively.

the basis of a common field in the full region with 80-100 figure accuracy.

A part of the E2 transition operator and the charge $Q_{k}$ of the $k$-th cluster is given by

$$
o_{k}=Q_{k}\left(3 z_{k}^{2}-x_{k}^{2}-y_{k}^{2}\right)=Q_{k} r_{k}^{2}\left(2 \cos ^{2} \theta_{k}-\sin ^{2} \theta_{k}\right) .
$$

However, in our simple S-wave calculation, the value of $\sum_{k=1}^{3}<\Psi_{f}\left|o_{k}\right| \Psi_{i}>$ becomes zero by the initial and the final wave functions $\Psi_{i}$ and $\Psi_{f}$. Therefore, we replace $o_{k}$ by $Q_{k} r_{k}^{2}=Z_{k} e r_{k}^{2}$. The calculated results for the $\mathrm{E} 2$ transition is represented by

$$
\begin{aligned}
& W_{i \rightarrow f}^{E 2}\left(E_{i}, E_{f}\right)=\frac{1}{20} \frac{4\left(E_{i}-E_{f}\right)^{5}}{3 \pi \varepsilon_{0}(h / 2 \pi)^{6} c^{3}} \sum_{k=1}^{3}\left|<\Psi_{f}\right| \frac{1}{2}\left(3 z_{k}^{2}-x_{k}^{2}-y_{k}^{2}\right) Z_{k} e\left|\Psi_{i}>\right|^{2} \\
& \rightarrow W_{i \rightarrow f}^{E 2^{\prime}}\left(E_{i}, E_{f}\right)=\frac{1}{20} \frac{4\left(E_{i}-E_{f}\right)^{5}}{3 \pi \epsilon_{0}(h / 2 \pi)^{6} c^{3}} \sum_{k=1}^{3}\left|<\Psi_{f}\right| \frac{1}{2} Z_{k} e r_{k}^{2}\left|\Psi_{i}>\right|^{2} .
\end{aligned}
$$

Therefore, the transition time is given by the inverse of $W_{i \rightarrow f}^{E 2^{\prime}}\left(E_{i}, E_{f}\right)$.

Finally, we obtained the ratio between $W_{i \rightarrow f}^{E 2^{\prime} ; L}\left(E_{i}, E_{f}\right)$ of the transition probability by the long range plus short range nuclear potential and $W_{i \rightarrow f}^{E 2^{\prime} ; S}\left(E_{i}, E_{f}\right)$ of the short range nuclear potential only,

$$
\frac{W_{i \rightarrow f}^{E 2^{\prime} ; L}\left(E_{i}, E_{f}\right)}{W_{i \rightarrow f}^{E 2^{\prime} ; S}\left(E_{i}, E_{f}\right)}=\frac{1.5 \times 10^{16} / S}{1.1 \times 10^{8} / S} \approx 10^{8}
$$

with $S=\mathrm{cm}^{2}$. The value of $W_{i \rightarrow f}^{E 2^{\prime} ; L}\left(E_{i}, E_{f}\right)$ is one order larger than the experimental value $\left(\sim 10^{15} / \mathrm{cm}^{2}\right)$ by Iwamura et al. for the ${ }^{133} \mathrm{Cs}+4 \mathrm{~d} \rightarrow{ }^{141} \mathrm{Pr}$, although our calculated result 
indicates the reaction: ${ }^{135} \mathrm{Cs}+2 \mathrm{~d} \rightarrow{ }^{139} \mathrm{La}$. Therefore, we conclude that the long range potential is essential to represent the experimental value.

\section{Conclusion and Discussion}

This work is based on our hypothesis of the long range GPT potential which is introduced by the three-body Faddeev method [15]. The GPT potential could emerge for the twobody form factor in the AGS Born term, because the two-body problem could be treated again by a certain three-body problem. Therefore, the long range effects in the three-body equation can not be written in terms of the sum of two-body long range potentials, but must be given by a three-body force-type or a nonlinear type potential. This is essentially predicted by Efimov in his first paper which we call the "long range three-body Efimov potential" based on the GPT potential, although several parameters of the potential are unfortunately not well defined.

In order to explore a possibility of the long range force, we discussed the nuclear synthesis at the room temperature. Evidence of the low energy nuclear synthesis was reported by Iwamura et al. in 2002 [7] and also in 2015 [16]. They measured the quantity of Cs and $\operatorname{Pr}$ by the XPS (X-ray photoelectron spectrometer) without taking out of the vacuum chamber, or in the Pd-cage where they measured unusual thermal release, but not observed the $\gamma$-ray emission. After the deuteron permeation, they etched the surface of Pd complex by using the $\mathrm{HNO}_{3}$. The etched solutions or the ions without the Pd-cage were measured by ICPMS (inductively coupled plasma mass spectrometry) etc., and finally the Pr element was confirmed by the mass. In the Pd-cage, if the reaction does not release sufficient energy, it is imagined that the element Pr does not go down to the nuclear ground state, but get stuck at a "stable excited nucleus Pr* state" between the molecular state and the nuclear ground state. If we remove the $\mathrm{Pd}$-cage by using the $\mathrm{HNO}_{3}$, the bare $\mathrm{Pr}^{*}$ ion should release the inner energy, and emit the $\gamma$-ray to go down to the ground state. Unfortunately, the experimentalists did not mention whether such an emission was observed.

Finally, it should be mentioned that the most important improvement in our calculation method is that the whole region from $0.01 \mathrm{fm}$ to several nano meters in the three-body equation is solved in a straight forward way with a very accurate method providing 100 digits. Furthermore, we found that the wave functions oscillate very quickly inside the potential barrier instead of being damped exponentially. Therefore, our calculated wave functions give non-zero overlapping values between the molecular states and the proper nuclear states even for the case without the long range potential, not to mention for the case with the GPT potential which is accompanied by some additional states.

In any case, our calculated result indicates that a different reaction rate should occur without the long range force and with the long range force.

We believe that our calculation is the first serious work for room temperature nuclear synthesis based upon the Faddeev approach using a long range potential [14].

\section{Acknowledgements}

The authors would like to acknowledge valuable discussions with Drs. N. Watari, H. Kakigami, N. Hamada and Y. Fukumoto regarding the theoretical aspects of the molecular system, as well as I. Toyoda, and S. Tsuruga for sharing with us their experimental insight. We are indebted to MHI Innovation Accelerator LLC Co. Ltd. for significant financial support. 


\section{References}

[1] Oryu S., Universal structure of the three-body system, Phys. Rev. C86, 044001 (2012).

[2] Oryu, S. A New Feature of the Efimov-Like Structure in the Hadron System: Long-Range Force as a Recoil Effect, Few Body Syst. 59(4), 51 (2018), doi:10.1007/s00601-018-1373-z.

[3] Nicholson, A. F., Bound states and Scattering in an $1 / r^{2}$ potential, Australian J. Phys. 15 174-179 (1962).

[4] Efimov V, Energy levels arising from resonant two-body forces in a three-body system, Phys. Lett. B33 563 (1970).

[5] Efimov V, Energy levels of three resonantly interacting particles, Nucl. Phys. A210 $157(1973)$

[6] Oryu S., Watanabe T., Hiratsuka Y., Takeda M., and Togawa Y., , Proceedings of FB22 Caen France (2018),

[7] Iwamura, Y., Sakano, M., and Itoh, T., Elemental Analysis of Pd Complexes: Effects of D2 Gas Permeation, Jpn. J. Appl. Phys., 414642 (2002).

[8] Hioki, T. et al., Inductively Coupled Plasma Mass Spectrometry Study on the Increase in the Amount of Pr Atoms for Cs-Ion-Implanted Pd/CaO Multilayer Complex with Deuterium Permeation Jpn. J. Appl. Phys., 52107301 (2013).

[9] Alt, E. O., Grassberger, P., and Sandhas, W., Reduction of the three-particle collision problem to multi-channel two-particle Lippmann-Schwinger equations, Nucl. Phys. B2 (1967) $167-180$.

[10] Oryu S.: Two- and three-charged particle nuclear scattering in momentum space: A two-potential theory and a boundary condition model, Phys. Rev. C73, 054001 (2006); Erratum: Phys. Rev. 76069901 (2007).

[11] Watanabe T., Hiratsuka Y., Oryu S., Togawa Y., A New Feature of the Screened Coulomb Potential in Momentum Space, Few-Body Syst. DOI 10.1007/s00601-0171238-x (2017).

[12] Oryu S.,Watanabe T., Hiratsuka Y. and Togawa Y., A Coulomb-Like Off-Shell TMatarix with the Correct Coulomb Phase Shift, Few-Body Syst. DOI 10.1007/s00601017-1258-6 (2017).

[13] Watari, N., Ohnishi, S. and Ishii, Y., Hydrogen storage in Pd-clusters, J. Phys:Condens. Matter 12 6799-6823 (2000).

[14] Oryu, S . An Investigation of the Nuclear Reaction Near the Three-Body Breack-up Threshold: As a Ultra Low Energy Nuclear Synthesis Few-Body Syst. 60-42 (2019) doi:.org10.1007/s00601-019-1508-x.

[15] Faddeev L D: Scattering theory for a three-particle system Zh. Eksp. Theor. Fiz. 39 1459-1467,(1960), Sov. Phys. -JETP 12 1014-1019, (1961).

[16] Iwamura, Y., Itoh, T., Tsuruga, S., Transmutation reactions induced by deuterium permeation through nano-structured palladium multilayer thin film, Current Science 108, 628-632 (2015). 